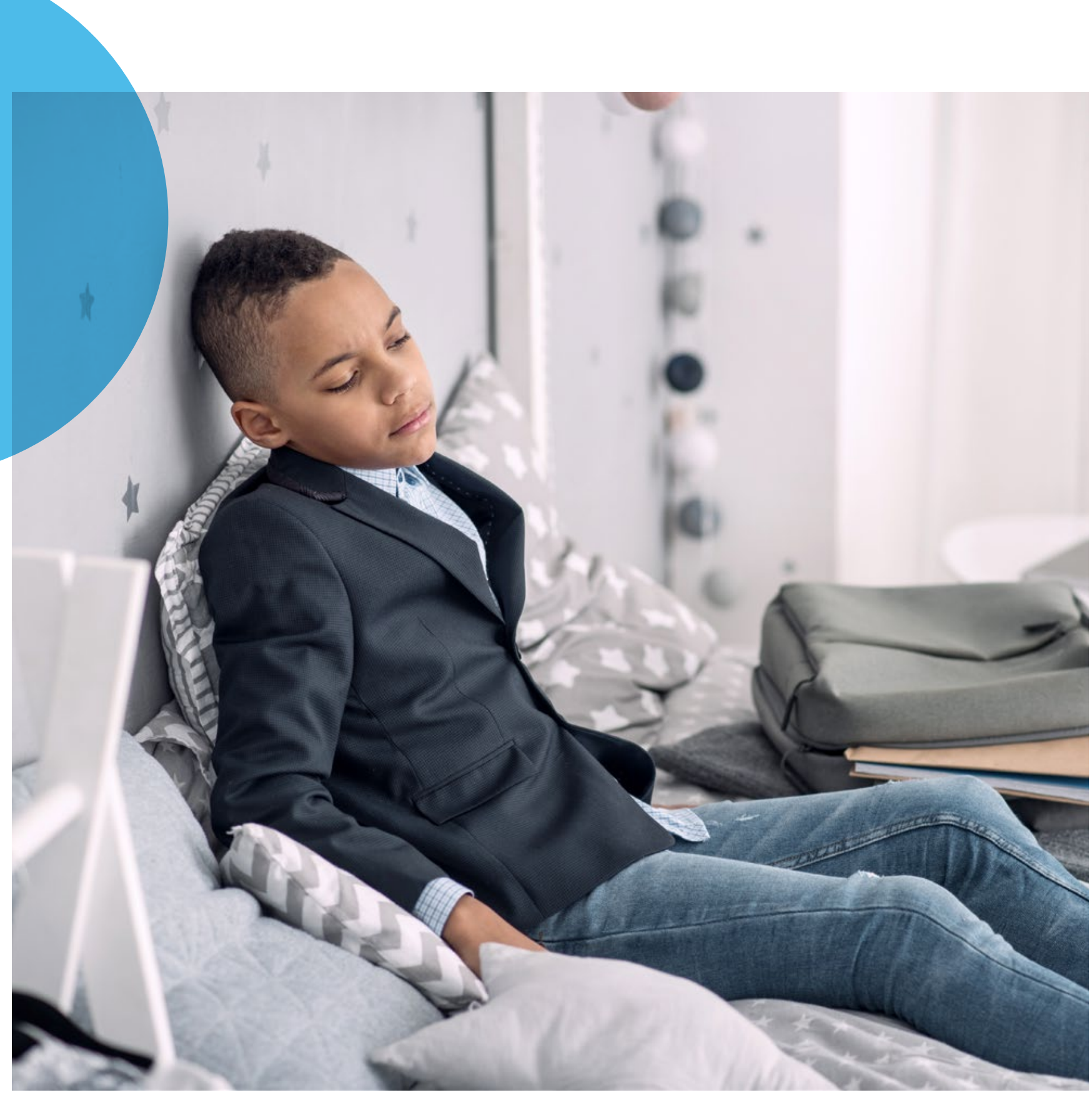

\title{
Determining the "IMPACT" of therapeutics for depression requires an adaptive trial design
}

By Dr Jessica K Edwards

A large proportion of adolescents suffering from moderate-to-severe major depression respond to psychological and pharmacological therapy, and the range of effective treatment modalities is increasing. Now, lan Goodyer and Paul Wilkinson have compiled a Practitioner Review that compares the various treatment options available and assesses their effectiveness for adolescents affected by major depressive episodes. 
In 2010, Professor lan Goodyer and Dr. Paul Wilkinson together with their colleagues around the UK launched the Improving Mood with Psychoanalytic and Cognitive Therapies (IMPACT) study ${ }^{1}$ to determine the costeffectiveness, safety and relapse rates of three treatment regimens known to reduce depressive symptoms. Specifically, they compared cognitive behavioural therapy (CBT) and short-term psychoanalytic psychotherapy (STPP) delivered over 5-7 months by specialists trained in these modalities, with a novel reference treatment of brief psychosocial intervention (BPI) delivered at low intensity within 3 months by psychiatrists and mental-health nurses. In 2017, the first published results from the IMPACT study indicated that neither CBT nor STPP were superior to each other, and that BPI was equivalent to both in maintaining reduced self-reported depression symptoms 1 year after treatment completion ${ }^{2}$.

In their 2018 Practitioner Review for the Journal of Child Psychology and Psychiatry, Goodyer and Wilkinson assessed the current state of knowledge on treatment modalities for major depressive episodes in adolescents and how the published data compared with the key findings of the IMPACT study - that BPI may be as good as accepted specialist CBT therapy or STPP. Their findings predominantly derive from four key randomized controlled trials (RCTs) that report on psychological and pharmacological treatments for depressed adolescents, and a set of studies that looked at prevention of the onset of major depressive disorder.

First focusing on psychological therapies, Goodyer and Wilkinson found that although promising results from RCTs have favoured CBT and interpersonal psychotherapy (IPT), a recent meta-analysis found their effect size to be relatively small (0.29) compared to active control groups 3 . The reasons behind this low effect size may be because of improvements in methodology and better active control treatments since the first RCTs of CBT and IPT were established. By contrast, another meta-analysis did find CBT and IPT to be efficacious and clinically effective in adolescents with depression. The researchers of both these meta-analyses noted that the number of IPT studies and sample sizes are too low to draw firm conclusions.

The IMPACT study, however, was sufficiently powered to examine whether specialist therapies exert therapeutic gains over those expected from a relatively simple active approach (BPI) delivered via a manual. "The results of IMPACT were thus surprising and in stark contrast to previous opinion in showing that all three psychological treatments (CBT, STPP and BPI) had potentially equal clinical effectiveness at the end of treatment and at 12-months follow-up", explains Goodyer. Early evidence indeed suggested that BPI may be effective for mild-to-moderate depression and anxiety disorders in adolescents. Now, further work must assess the effectiveness of the BPI method used in the IMPACT study in a community, rather than a clinical setting.
Goodyer and Wilkinson then turned their attention to the therapeutic effects elicited by antidepressants. Here, they found that the most robustly designed studies showed that antidepressants can achieve an efficacy up to $20-25 \%$ higher than placebo ${ }^{4}$. Some selective serotonin reuptake inhibitors (SSRIs), most notably fluoxetine, elicit strong effects over placebo, but other antidepressants (e.g. mirtazapine and venlafaxine) have no proven effects. Furthermore, data suggest that SSRIs may increase suicidal thinking in some adolescents with depression: in rare cases, fluoxetine can induce disinhibition and switching to mania. More trials that recruit patients with severe depression are needed to deeply evaluate SSRI efficacy and determine any potential adverse effects.

Goodyer and Wilkinson next assessed treatment response, treatment non-response and relapse for the aforementioned therapeutic approaches. Response rates in treatment-naïve adolescents with depression are usually fairly rapid, with up to $30 \%$ reduction in symptoms achievable in the first 12 weeks of starting some form of treatment. Remission rates are also high, with reportedly $80 \%$ of trial patients remaining in clinical remission (no diagnosis or persistent $50 \%$ reduction in baseline symptoms) for up to 1 year after treatment end.

\section{"up to $30 \%$ reduction in symptoms achievable in the first 12 weeks of starting some form of treatment"}

Unfortunately, relapse rates are high (50-75\%) in successfully treated patients. As such, Goodyer and Wilkinson recommend that clinical monitoring should be continued for an additional 6 months after treatment, or until at least a 50\% drop in symptoms is reached. They also suggest that "booster" psychological treatment sessions could be offered to those showing early signs of relapse.

In those who do not show any initial response to treatment (up to $40 \%$ adolescents), a combination treatment strategy should be recommended that employs both psychological and pharmacological treatment modalities. "Treatment is effective, be it psychological or pharmacological, for about two thirds of patients", reinforces Goodyer. "Clinicians should make good assessments and choose collaboratively with the patient what treatment to use. There is no reason to not treat all patients".

Although no demographic factors have been identified that can reliably predict these described differences in treatment outcome, clinical severity at baseline may be associated with a poor treatment response. Parameters at first assessment associated with clinical severity of depression include poor global functioning, high levels of suicidality, co-morbid anxiety, cognitive distortions, hopelessness and family conflict. 
Moderators of therapeutic change have been identified in many of the large RCTs. For example, combining fluoxetine treatment with CBT in the TADS trial resulted in improved outcomes in patients with mild-to-moderate depression but not in those with severe depression compared to either single treatment alone. In the TORDIA study of adolescents with treatment-resistant depression, adding CBT to SSRI treatment was effective in those with more co-morbid disorders.

The researchers consider that a strong step forward in the field will be achieved through the development of adaptive clinical trials for depression therapeutics. Such trials can make prospectively planned changes to the course of the trial based on the accumulating data from the trial itself ${ }^{5}$. In the context of confirming effectiveness of therapeutics in depression, Goodyer and Wilkinson propose that one such adaptive trial could start with treatment in phase one (e.g. BPI), and progress to phase two (e.g. CBT) only if symptoms fail to reduce from baseline by $>50 \%$. The second phase can then progress to phase three (e.g. combined fluoxetine and CBT) if symptoms still fail to reduce by $>50 \%$ from baseline. If at any phase, symptoms are $<50 \%$ of baseline, the trial can stop as remission would be achieved.

Although more complex than classical RCTs that deliver a single treatment modality - especially with regards to the statistical techniques and procedures needed to ensure the data are free from bias - adaptive trials can help overcome common limitations such as the need for a large sample size and a long study duration, as well as lack of power and high costs ${ }^{6}$.

In summary, Goodyer and Wilkinson note that while acknowledging prevention is better than a cure, there are currently no clear strategies that have been identified to reduce the incident onset of depression in this population. However the data so far does indicate that major depressive episodes are highly treatable, with active therapies being more effective than active placebos alone. The authors explain that knowledge is still lacking as to how any treatment works and what treatment works for whom. Studies that aim to identify new medications and/or psychological therapies for severely treatment-resistant cases are also urgently needed. Ultimately, they consider that a precision medicine approach needs to be adopted for adolescents with depression that is tailored to the needs of the individual patient. "Going forward scientifically, we recommend that adaptive trials be established that will disaggregate what treatment works for which subtype of depression", says Goodyer. "It is a complex design, but will help answer that key question better than any trial has done to date".
Referring to:

Goodyer, I. M. \& Wilkinson, P. O. (2018), Practitioner Review: Therapeutics of unipolar major depressions in adolescents. J Child Psychol Psychiatr. doi:10.1111/jcpp.12940

\section{Further reading:}

${ }^{1}$ Goodyer, I. M. et al. (2011) Improving mood with psychoanalytic and cognitive therapies (IMPACT): a pragmatic effectiveness superiority trial to investigate whether specialised psychological treatment reduces the risk for relapse in adolescents with moderate to severe unipolar depression: study protocol for a randomised controlled trial. Trials (12) 125. doi: 10.1186/1745-6215-12-175.

${ }^{2}$ Goodyer, I. M. et al. Cognitive behavioural therapy and short-term psychoanalytical psychotherapy versus a brief psychosocial intervention in adolescents with unipolar major depressive disorder (IMPACT): a multicentre, pragmatic, observer-blind, randomised controlled superiority trial. Lancet Psychiatry. 2017; 4: 109-11. doi: 10.1016/ S2215-0366(16)30378-9.

${ }^{3}$ Weisz, J. R. et al. (2017). What five decades of research tells us about the effects of youth psychological therapy. A multilevel meta-analysis and implications for science and practice. American Psychologist, 72: 79-117. doi: 10.1037/a0040360.

${ }^{4}$ Walkup, J. T. (2017). Antidepressant efficacy for depression in children and adolescents: industry-and NIMH-funded studies. American Journal of Psychology, 174: 430-437. doi: 10.1176/appi.ajp.2017.16091059.

${ }^{5}$ Bhatt, D. L. et al. (2016). Adaptive designs for clinical trials. New England Journal of Medicine, 375: 65-74. doi: 10.1056/NEJMra1510061.

${ }^{6}$ Thorlung, K. et al. (2018). Key design considerations for adaptive clinical trials: a primer for clinicians. The British Medical Journal, doi: 10.1136/bmj.k698.

\section{Glossary:}

Cognitive behavioural therapy (CBT): a form of talking therapy that encourages patients to manage their psychosocial problems by changing the way they think and behave; CBT focuses on current problems and finds practical ways to improve state-of-mind on a day-by-day basis

Short term psychodynamic psychotherapy (STPP): a manualized psychoanalytic treatment for depression in adolescents aged 13-19, which involves 28 sessions for the patient and up to 7 sessions for his/her parents or carers

Brief psychosocial intervention: interpersonal or informational activities or strategies that aim to improve health functioning and well-being. BPI is built on three core principles: (1) a collaborative approach plus psychoeducation; (2) selected use of behavioural activation techniques; and (3) instigation of recovery support methods

Randomised controlled trial: an experimental setup whereby participants are randomly allocated to an intervention/treatment group or a control/placebo group; randomization of participants occurs after assessments for eligibility, and is used to minimize selection bias

Interpersonal psychotherapy: brief, attachment-focused therapy for those with mood disorders that aims to resolve interpersonal problems and promote symptomatic recovery

Selective serotonin reuptake inhibitor (SSRI): a commonly prescribed antidepressant that works by increasing the levels of serotonin in the brain by selectively blocking serotonin reabsorption

Adaptive clinical trial: a trial that permits continued modification to the trial design based on accumulating, interim data 